\title{
Discrete internal pattern representations and visual detection of small changes in pattern shape
}

\author{
DAVID H. FOSTER \\ Department of Communication and Neuroscience \\ University of Keele, Keele, Staffordshire ST5 5BG, England
}

\begin{abstract}
A scheme is proposed that relates the internal representation of pattern stimuli to visual pattern discrimination performance. The main assumptions of the scheme are: (1) internal representations are assigned to patterns probabilistically; (2) internal representations are discrete; (3) visual discriminability of two patterns is determined by the differences in the assignment probabilities of their internal representations. It is suggested that the internal representations associated with a pattern may be analyzed by measuring the visual detectability of small changes in pattern shape at points along a continuum of shapes. This continuum is generated by a group of transformations smoothly parameterized by a single variable. In two experiments designed to test this approach, subjects discriminated pattern displays containing combinations of three-dot subpatterns. Under the hypothesis that there were just two relevant internal representations, specifying dot collinearity and dot noncollinearity, the qualitative characteristics of the discrimination performance were correctly predicted.
\end{abstract}

An object or pattern presented to the visual system may be thought of as producing an internal representation which, in turn, provides a basis for the memorization of the stimulus and the generation of a response. This representation is often assumed to be an encoding that selectively specifies the structural properties of the stimulus. One way to interpret this structure is to consider the internal representation as a collection of various pattern attributes that includes global features like symmetry and area, local features like points, lines, and regions, and spatial relations between these local features such as "left of," "joined to," and "inside of." A representation consisting jointly of local features and spatial relations is sometimes called a structural description (Barlow, Narasimhan, \& Rosenfeld, 1972; Sutherland, 1973). Particular versions of internal representations involving local features or combinations of local features and relations have been proposed by, amongst others, Foster and Mason (1979), Gibson (1969), Marr (1976), Palmer (1978), Reed (1974), Sutherland (1968), and Rumelhart (Note 1). An alternative approach to the formalization of the structure of internal representations has been discussed by Foster (1975, 1977). In terms of information processing schemes (Broadbent 1971; Reed, 1973; Turvey, 1978), internal representations may be assumed to be constructed at some

I thank D. M. Mackay and D. P. Andrews for stimulating discussions, R. Knapper for technical assistance, and S. Gravano, J. I. Kahn, R. J. Mason, and R. J. Watt for critical readings of an earlier version of this report. point after formation of the hypothesized internal visual "image" or icon (Neisser, 1967; Sperling, 1963).

Whatever its form, the internal representation of a given pattern is clearly not fixed under all conditions, for, apart from its decay following removal of the physical stimulus, the extent of the faithfulness with which the internal representation describes the pattern will generally depend on the conditions of presentation and on the set of the observer. For example, a letter "o" with an appropriately small gap in the right side may be identified either as an "o" or as a "c," bias to one or other of these responses being influenced by the size of the gap and the context of the ambiguous character (Blesser, Shillman, Cox, Kuklinski, Ventura, \& Eden, 1973). Further examples are provided by the classical ambiguous figures like the Necker cube. In general, the assignment of an internal representation to a pattern may be considered to be a probabilistic process.

Suppose internal representations are, in fact, composed of combinations of components drawn from some set of pattern attributes (such as local features and spatial relations), and suppose further that this set is finite. The population of all possible internal representations generated by this method is infinite, like the vocabulary of words generated by an alphabet. Despite the number of internal representations potentially available, there are limitations in the accuracy with which they can reflect small changes in the shape of pattern stimuli. The most important property of the population, however, 
derives from the combinatorial nature of its construction. The internal representations produced in this way are discrete.

If visual discrimination of patterns were determined by discrete internal representations, then one might expect to observe discontinuities in performance analogous to those reported in speech perception. The discrete representations in this case would correspond to the phoneme categories. (This analogy is suggested only at a formal level.) Liberman, Harris, Hoffman, \& Griffith (1957) showed that as certain speech-like stimuli are varied in small steps along an acoustic continuum, adjacent pairs of stimuli are discriminated better when they fall on different sides of a phoneme boundary than when they fall within the same phoneme category (see Liberman, Harris, Kinney, \& Lane, 1961; Massaro, 1976; Pisoni \& Lazarus, 1974). For visual pattern discrimination, the situation is more complex, since there are no natural dimensions (like voice onset time) along which the shape of the stimulus may be progressively altered. This difficulty is circumvented here by constructing a continuum of pattern shapes and steps along that continuum by means of a group of spatial transformations, smoothly parameterized by a single variable.

In this study, a scheme is put forward in which patterns are internally encoded in terms of discrete representations which are assigned probabilistically. It is assumed that the visual discriminability of patterns is then determined by differences in these assignment probabilities. Making use of this assumption, a technique for the analysis of these internal representations is proposed. To provide an illustration and test of the technique, some experiments are performed which entail the discrimination of various arrangements of simple dot patterns. The qualitative characteristics of the measured discrimination performance are predicted on the basis of plausible estimates of the internal representations associated with the patterns.

\section{DISCRETE PROBABILISTIC REPRESENTATIONS AND VISUAL DISCRIMINATION}

The basic assumptions of the scheme for internal representations are listed below. Certain technical conditions need also to be satisfied, but in practice they are unlikely to be restrictive and are given in Appendix 1.

(1) Probabilistic assignment. For a given stimulus pattern, A, there are a finite number of distinct internal representations, $r_{1}, r_{2}, \ldots, r_{k}$, produced by A with corresponding assignment probabilities, $p_{1}(A)$, $\mathrm{p}_{2}(\mathrm{~A}), \ldots, \mathrm{p}_{\mathrm{k}}(\mathrm{A})$, which sum to unity. On each presentation, it is certain that at least one of the internal representations, $r_{i}$, is assigned to the pattern and not more than one representation is assigned at a time.

(2) Discreteness. For every pattern, A, there exists a finite collection of available internal representations which includes all the internal representations that $\mathrm{A}$ produces and, in addition, for sufficiently small smooth perturbations in the shape of $A$, all the internal representations produced by these perturbed versions of $A$. This is the discreteness property mentioned in the introduction.

(3) Discrimination by probability differences. Performance in visually discriminating two patterns, $A$ and $B$, is determined by the differences in the assignment probabilities, $\mathrm{p}_{i}(\mathrm{~A})$ and $\mathrm{p}_{\mathrm{i}}(\mathrm{B})$, of the internal representations, $r_{i}, i=1,2, \ldots, n$, generated by the two patterns; specifically, discriminability increases monotonically with these differences. (Metrics for evaluating these differences are given in Appendix 1.) For example, if the probabilities $p_{j}(A)$ and $p_{m}(B), j \neq m$, are each close to unity, then the visual discriminability of $A$ and $B$ will be higher than if $p_{j}(A)$ and $p_{j}(B)$ are each close to unity. In the first case, the internal representations assigned to $A$ and $B$ are almost always different, and in the second case, they are almost always the same.

Suppose one applies to a pattern, A, a group of spatial transformations smoothly parameterized by some variable, s (see Appendix 1). As $s$ is varied, the probabilities, $\mathrm{p}_{\mathrm{i}}(\mathrm{A})$, associated with the representations, $r_{i}$, also vary. Figure 1 shows how the probabilities of three representations, $r_{1}, r_{2}$, and $r_{3}$, might depend on s. For values of $s$ around point a, representation $r_{1}$ is almost always assigned; similarly, for values of $s$ around point $c, r_{2}$ is almost always assigned, and for values of $s$ around point $e, r_{3}$ is almost always assigned. If $s$ is incremented by small fixed amounts, $+\Delta s$ and $-\Delta s$, about some reference point, how detectable are the corresponding changes

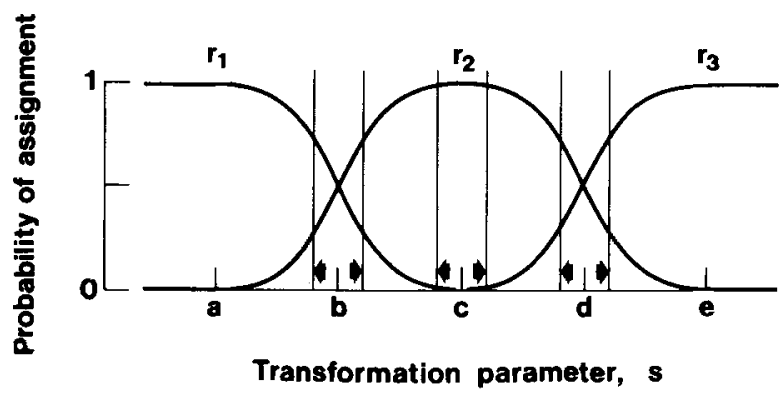

Figure 1. Hypothetical dependence of assignment probabilities on pattern transformation parameter $s$ for three internal representations $r_{1}, r_{2}$, and $r_{3}$. Increments in $s$ of $-\Delta s$ and $+\Delta s$ about points $b$, $c$, and $d$ are indicated by the pairs of arrowed vertical lines. 
induced in the pattern? At a point such as c, the assignment probabilities undergo very little change as the pattern is transformed from state $s-\Delta s$ to state $s+\Delta s$ (indicated in the figure by the arrowed pair of vertical lines about $c$ ). In contrast, at a point such as $\mathrm{b}$ or $\mathrm{d}$, there are large and opposite changes in the assignment probabilities as the pattern is transformed from state $s-\Delta s$ to $s+\Delta s$ (again indicated by the arrowed lines). The prediction of Assumption 3 above is that the visual detectability of these pattern changes is low at point $c$, and at points a and $e$, and high at points $b$ and $d$.

Provided that there are not more than effectively two competing internal representations at each value of the transformation parameter $s$, it may be shown that this result concerning the discrimination of patterns holds for more general distributions of internal representations (Appendix 1). Thus, where assignment probabilities are maximum, discrimination performance should be minimum, and where assignment probabilities are changing most rapidly, discrimination performance should be maximum. This relationship is the basis of the suggested technique for investigating the internal representations of the scheme formalized above.

It is not necessary to know the precise probabilities of the internal representations produced by a pattern and a group of transformations in order to attempt a test of the technique. If one can estimate only the value of the transformation parameter at which some internal representation has maximum probability of assignment, certain qualitative characteristics of the discrimination performance are still implied. This is the situation examined in the experiments described below.

The internal representation considered here defines one of the basic quantities interrelating stimulus elements, namely, the collinearity of those elements. There have been a number of studies which have led to the suggestion that collinearity or its failure has a special role in visual function, for example, in perceptual grouping (Prytulak, 1974; Wertheimer, 1923), in visual detection (Caelli \& Umansky, 1976; Prinzmetal \& Banks, 1977; Uttal, 1973), and in visual acuity (Andrews, Butcher, \& Buckley, 1973; Bouma \& Andriessen, 1968). The simplest stimulus that can specify collinearity or noncollinearity is a figure consisting of three points. The stimulus patterns used in the present experiments thus consisted of collections of three-dot subpatterns each of the form illustrated in Figure 2. The parameter s measures the angle between the lines defined by dots 1 and 3 and by dots 1 and 2 , the distances between dots 1 and 2 and between dots 2 and 3 being constant. The task of the subject entailed the detection of subpattern changes produced by fixed increments $\pm \Delta s$ in the angle $s$, as $s$ varied over a range of values. This last condi-

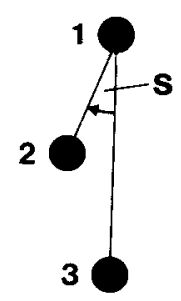

Figure 2. Stimulus subpattern. The center-to-center distances between dots 1 and 2 and between dots 2 and 3 are constant and equal, each subtending $\mathbf{0 7 5}$ deg at the eye. The deformation of the subpattern is specified by the parameter $s$, which measures the planar angle shown (positive clockwise). The dots appeared white against a uniform background.

tion and the number of dot subpatterns in a display distinguishes the present experiments from some vernier-acuity studies also using simple combinations of pattern elements (Beck \& Schwartz, 1979; Ludvigh, 1953; Westheimer \& McKee, 1977).

The logic of the experiments is this. When the deformation angle $s$ is zero, the dots in the subpattern are in line, and by hypothesis give rise with high probability to an internal representation which specifies (amongst other things) the collinearity of the dots. As $s$ increases in magnitude, some other representation specifying the noncollinearity of the dots is assumed to become more probable. If the arguments set out above are correct, then visual detectability of the pattern perturbations caused by the increments $\pm \Delta$ s should be minimum about the reference value $s=0^{\circ}$, and as $s$ increases positively or negatively from this point detectability should eventually reach a maximum, and then decrease again. This form of pattern discrimination performance would not necessarily be expected on the basis of considerations applied to the interpretation of vernier-acuity experiments.

\section{EXPERIMENT 1}

A two-interval forced-choice procedure was used to measure the discriminability of perturbed patterns from unperturbed patterns. Figure 3 shows to scale a typical pair of displays. In Figure 3a, the left and right halves of the field are different in that the top two subpatterns are each perturbed in opposite directions (corresponding to transformation parameter values $s-\Delta s$ and $s+\Delta s$ ); in Figure $3 b$, the left and right halves of the field are the same. ${ }^{1}$ The task of the subject was to select the "different" display.

\section{Method}

Stimuli and Apparatus. The dot patterns were produced online by computer on the screen of an X-Y display oscilloscope (Hewlett-Packard, Type 1300A) with a P4 sulfide phosphor. The screen was viewed binocularly at $1.7 \mathrm{~m}$ through a view tunnel 


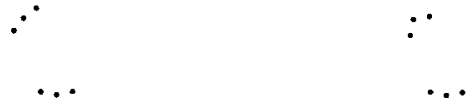

(a)

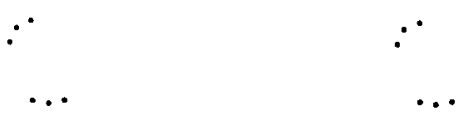

(b)

Figure 3. Example of stimulus patterns used in a two-interval presentation in Experiment 1. In display a, the left and right halves of the field are different in that the top two subpatterns are perturbed in opposite directions; in display $b$, the left and right halves are the same.

and optical system which produced a uniform background field upon which the dot patterns appeared superimposed. ${ }^{2}$ A small central fixation spot was present before and after, but not during, the two-interval presentation period.

Each dot in the display subtended about $.02 \mathrm{deg}$ at the eye, and the constant center-to-center gap between the center dot (dot 2 in Figure 2) and each of the two outer dots (dots 1 and 3) in each subpattern subtended $.075^{\circ} .{ }^{3}$ The positions of the perturbed and unperturbed subpatterns were chosen pseudorandomly, but were constrained so that on each side the subpatterns were located within a circle of radius $.3 \mathrm{deg}$ and separated by at least .3 deg. The centers of the two constraining circles were $.9 \mathrm{deg}$ horizontally to the left and to the right of the fixation point.

Procedure. The subject was informed that each trial would involve the presentation of two displays, the one containing the same patterns and the other containing different patterns. He was instructed to indicate, after the two presentations, the one in which the different pair occurred. The subjects (other than the author) were not informed of the nature of the differences in the patterns. The subjects were encouraged to respond as quickly as was consistent with accuracy.

The subject fixated the central fixation spot and, when ready, initiated a trial by pressing the appropriate switch on a pushbutton box interfaced to the computer. The fixation spot disappeared and after a 500 -msec blank field, the first display (e.g. as in Figure 3a) appeared for $80 \mathrm{msec}$; after a 1,500-msec blank field, during which the subject maintained central fixation, the second display (e.g., as in Figure 3b) appeared for $80 \mathrm{msec}$. When the subject had signaled his response on the push-button box, the fixation spot reappeared after about $2 \mathrm{sec}$ delay indicating that the next trial could be started.

Each run comprised 28 trials in which each subpattern had seven different reference values, $s$, of the transformation parameter (ranging from -30 to $+30 \mathrm{deg}$, see Figure 2), and four different orientations $(0,45,90$, and $135 \mathrm{deg}$ to the vertical). The magnitude of the increments $\pm \Delta s$ about $s$ was fixed (depending on the subject) at either 14 or $18 \mathrm{deg}$. Each subject carried out one preliminary practice run and then 12 or 24 runs over a period of several days. The sequence of presentations in each run was chosen pseudorandomly but balanced over runs to offset stimulus carry-over effects and response bias by the subject. No feedback was given concerning the correctness of the subject's response.
Subjects. There were four subjects, aged from 18 to 34 years: two female, F.M.F. and J.M., and two male, J.I.K. and D.H.F. (the author). All had had previous experience in performing psychophysical experiments with briefly presented pattern displays. Each had normal or corrected-to-normal vision (acuities each not worse than 6/5). F.M.F. and J.M. were unaware of the purpose of the experiment.

\section{Results}

Figure 4 shows the results of the "same-different" discrimination experiment. For each subject, the percentage of correct "different" responses, corresponding to a correct discrimination of perturbed patterns from unperturbed patterns, is plotted against the reference value, $s$, of the transformation parameter. Results are pooled over all perturbed subpattern orientations. The magnitude of the fixed increments, $\pm \Delta s$, in the transformation parameter is indicated (14 or $18 \mathrm{deg}$ ) against the subject's initials, and the number of trials performed at each value of $s$ is shown in parentheses. Schematic illustrations of the perturbed subpatterns with parameter values $s+\Delta s$ and $s-\Delta s$ are given below the abscissa.

Despite individual differences, it is obvious that each subject's discrimination performance is lower at reference value 0 deg than at adjacent values $( \pm 10$ and $\pm 20 \mathrm{deg}$ ). The vertical positions of the discrimination curves, which vary over subjects, depend on the values of $\Delta s$, is shown for subject F.M.F.

The data were analyzed formally by means of techniques for binary data analysis based on the use of the logistic transform $\log [\mathrm{p} /(1-\mathrm{p})]$ of the probability $\mathrm{p}$ (Cox, 1970, Sec. 3.4). Modified logistic transforms were computed and standardized contrasts then constructed (Appendix 2). The depressions in discrimination performance at $s=0$ deg relative to \pm 10 and \pm 20 deg were found ${ }^{4}$ to be highly significant $\left(\chi_{4}^{2}=38.2, \mathrm{p}<.001\right)$. The depressions at $\mathrm{s}=$ $\pm 30 \mathrm{deg}$ relative to $\pm 20 \mathrm{deg}$ are also significant $\left(\chi_{4}^{2}=9.8, p<.05\right)$, and so are the asymmetries in performance about $0 \mathrm{deg}\left(\chi_{4}^{2}=11.2, \mathrm{p}<.05\right)$. No subpattern orientation effect or any of the interactions between $s$ and subpattern orientation reached significance $\left(\chi_{4}^{2} \leqslant 8.9, \mathrm{p}>.05\right)$.

The form of the discrimination characteristics, namely, a minimum when the reference value $s=0 \mathrm{deg}$ and a maximum each side of this point, agrees with the prediction of the proposed representations scheme. ${ }^{5}$ There are, however, other possible interpretations of the data. For example, it might be hypothesized that although the terms "same" and "different" used in the instructions are neutral with respect to the parametric changes in the subpatterns, subjects may have construed these terms nonuniformly with those changes. Alternatively, it might be hypothesized that subjects judged patterns solely on the basis of their noncollinear subpattern components. Because each "different" display contained both perturbed and unperturbed subpatterns on each side (Figure 3a), this strategy could have reduced performance to 


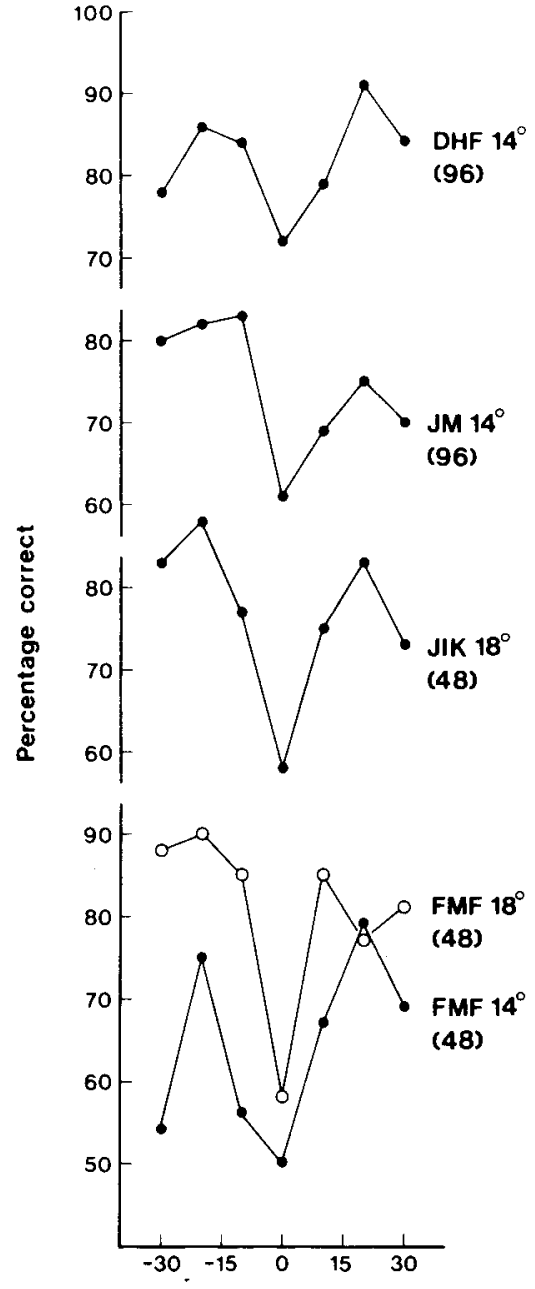

Reference value of transformation parameter, deg

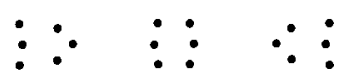

Figure 4. Discrimination of perturbed patterns from unperturbed patterns (Experiment 1). For each subject, the percentage of correct responses is shown as a function of the reference value $s$ of the transformation parameter. The number of trials performed at each point ( 48 or 96 ) and the magnitude of the fixed increments $\pm \Delta s$ in the transformation parameter are indicated against the subject's initials. Schematic illustrations of perturbed subpatterns with transformation parameters $s+\Delta s$ and $s-\Delta s$ are shown below the abscissa.

chance levels when reference value $s=0$ deg for the perturbed subpatterns. Another alternative hypothesis concerning the depression at $0 \mathrm{deg}$ is that it is a consequence of a general failure to distinguish mirror images; at $0 \mathrm{deg}$, the perturbed subpatterns have the same shape apart from being reflected about the long axis. These hypotheses were tested in the following experiment.

\section{EXPERIMENT 2}

Each trial involved a single presentation of a display consisting of four vertically oriented subpatterns placed approximately symmetrically about the fixation point, as in Figure 5. In the display, all the subpatterns have the same reference value $s$ of the transformation parameter, but one subpattern (the top) is "odd" in that it is derived from a positive increment $+\Delta s$ about $s$, whereas the other three are derived from a negative increment $-\Delta \mathrm{s}$. The task of the subject, who was appraised of the nature of the pattern changes, was highly specific, namely to indicate the direction of perturbation for the odd subpattern.

\section{Method}

Stimuli and Apparatus. The dimensions of the subpattern stimuli and the method of their generation and presentation were the same as in Experiment 1. Each subpattern was located pseudorandomly within a circle of radius $.3 \mathrm{deg}$, centered .9 deg right of, left of, above, or below the fixation point.

Procedure. The subject was informed that in each display one of the subpatterns was odd in that its center dot was displaced relative to its top and its bottom dots more to the right or to the left than was the case for the other three (identical) subpatterns. He was instructed to indicate in which direction, left or right, the displacement for the odd subpattern occurred.

Apart from there being just one presentation in each trial, the time course of each trial and the general conditions of viewing followed those of Experiment 1. Each run comprised 28 trials in which there were seven different reference values, $s$, of the transformation parameter $(-30$ to $+30 \mathrm{deg})$, and four different general positions of the odd subpattern (left, right, top, and bottom). The magnitude of the increments $\pm \Delta s$ was fixed at either 10 or $14 \mathrm{deg}$. The subjects were given a preliminary practice run in which presentation times were increased to $4 \mathrm{sec}$ so that they could make a detailed inspection of the individual subpatterns. Each subject performed 12 runs, ordered and balanced as in Experiment 1 .

Subjects. There were four subjects: one female, F.M.F., and three male, J.I.K., R.J.M., and D.H.F. All but R.J.M. had participated in Experiment 1. R.J.M., aged 25 years, was familiar with the type of discrimination task involved and had correctedto-normal vision (acuity 6/4). F.M.F. was unaware of the purpose of the experiment. 


\section{Results}

The results of the perturbation-direction discrimination experiment are shown in Figure 6 by the continuous lines. (The broken lines relate to the auxiliary experiment discussed later.) For each subject, the percentage of responses correctly indicating the direction of odd-subpattern perturbation is plotted against the reference value, $s$, of the transformation parameter. Results are pooled over odd-subpattern positions. The magnitude of the fixed increments, $\pm \Delta \mathrm{s}$, in the transformation parameter (10 or $14 \mathrm{deg}$ ) and the number of trials at each value of $s$ are shown against each subject's initials.

As found in Experiment 1, there is a marked decrease in discrimination performance at $\mathrm{s}=0 \mathrm{deg}$, maxima in performance at \pm 10 and $\pm 20 \mathrm{deg}$, and a falloff in response at $\pm 30 \mathrm{deg}$. There are some differences, however, in the two sets of data. In the present experiment, high performance levels are achieved with smaller values of $\Delta s$, and some of the peaks in performance are sharper. (Subject J.I.K. used the smaller value of $\Delta \mathrm{s}$ here, in contrast to Experiment 1.) But, given the different structure of the two experiments, there is an evident stability in the shape of the discrimination characteristics.

A formal analysis was applied to the data by means of the same procedures as used in Experiment 1. The depressions in discrimination performance at $s=0 \mathrm{deg}$ relative to \pm 10 and \pm 20 deg were found ${ }^{4}$ to be highly significant $\left(\chi_{4}^{2}=41.4, \mathrm{p}<.001\right)$, as were the depressions at $\mathrm{s}= \pm 30$ deg relative to $\pm 20 \mathrm{deg}\left(\chi_{4}^{2}=\right.$ $42.5, \mathrm{p}<.001)$. The asymmetries about 0 deg also reached significance $\left(\chi_{4}^{2}=11.4, p<.05\right)$. Oddsubpattern location gave a highly significant effect for the top vs. bottom position $\left(\chi_{4}^{2}=22.0, p<.001\right)$. The only significant interaction was between the relative depressions at $s= \pm 30 \mathrm{deg}$ and the leftright vs. top-bottom subpattern positions $\left(\chi_{4}^{2}=12.5\right.$, $\mathrm{p}<.05$ ).

In view of the agreement between the form of the data for this experiment and Experiment 1, it seems reasonable to reject the interpretations of the results based on methodological factors mentioned earlier. It was, however, suggested, in the discussion of Experiment 1, that the drop in performance at $s=$ 0 deg might be due to a general failure of subjects to discriminate mirror images. Because of the arrangement of the subpatterns in the field, the second experiment is more vulnerable to this objection than the first. To clarify the role of mirror-image confusion in determining discrimination performance, two control measurements were made. The first determined if there exists, as the proposed scheme implies, a reduction in discrimination performance at small $s$ values where perturbed subpatterns are not mirror images. For subject J.I.K. (Figure 6), this is already seen to be true, but for the other subjects, the scale for $s$ is too coarse to resolve the issue. The second

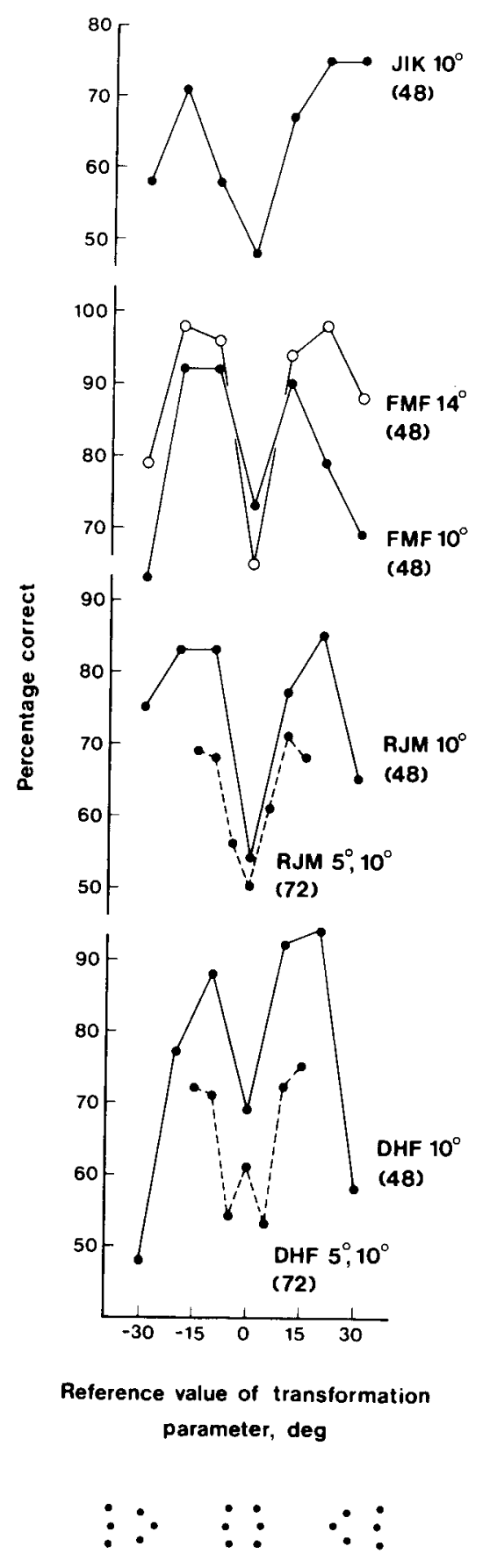

Figure 6. Discrimination of direction of odd-subpattern perturbation (Experiment 2). For each subject, the percentage of correct responses is shown as a function of the reference value $s$ of the transformation parameter. The continuous lines show data for the main part of the experiment in which the magnitude of the increments, $\pm \Delta s$, in the transformation parameter is constant over all values of $s$; the broken lines show data for the auxiliary experiment in which the value of $\Delta s$ differs for $s=0$ deg and $s \neq 0$ deg. In each case, the value of $\Delta s$ and the number of trials at each point (48 or 72 ) are indicated against the subject's initials. Schematic illustrations of the odd subpattern and one of the three identical comparison subpatterns, with transformation parameters $s+\Delta s$ and $s-\Delta s$, are shown below the abscissa. 
control determined whether the subpatterns of the mirror-image perturbed pair are less discriminable from each other than each is from a collinear subpattern. Mirror-image confusion might be expected to give such a result. These two controls were carried out in the following experiment.

\section{Auxiliary Experiment}

Methods and procedure were the same as in the main part of Experiment 2, except that (1) pattern perturbations were made about reference value $s$ incremented in 5-deg steps $(-15,-10, \ldots, 15 \mathrm{deg})$, and (2) the magnitude of the increments $\pm \Delta s$ was $5 \mathrm{deg}$ for all $\mathrm{s} \neq 0 \mathrm{deg}$ but $10 \mathrm{deg}$ for $\mathrm{s}=0 \mathrm{deg}$.

The points connected by the broken lines in Figure 6 show the results of this experiment for two subjects, R.J.M. and D.H.F. For both subjects, discrimination performance is lower at $s= \pm 5 \mathrm{deg}$ than at \pm 10 and $\pm 15 \mathrm{deg}$ (for each, $z \geqslant 2.23, p<.05$ ). Performance at $\mathrm{s}= \pm 5 \mathrm{deg}$, where $\Delta \mathrm{s}=5 \mathrm{deg}$, relative to that at $s=0 \mathrm{deg}$, where $\Delta s=10 \mathrm{deg}$, is different for the two subjects, but not significantly so (for each, $|z| \leqslant 1.10, p>.1$.

The outcome of these controls is consistent with the proposed scheme. First, there is a reduction in discrimination performance for small $s$ values and non-mirror-image subpatterns. Second, if there is an effect in the main experiments due to mirror-image confusion, it is not important in determining the shape of the discrimination characteristics.

\section{SUMMARY AND DISCUSSION}

The scheme for internal representations proposed here has three main assumptions. First, internal representations are assigned to a pattern on a probabilistic basis. Second, internal representations are discrete. Third, the visual discriminability of two patterns is determined by the differences in the assignment probabilities of the internal representations associated with each pattern.

For such a scheme, it is suggested that the internal representations associated with a pattern may be analyzed by a technique which involves making small changes in pattern shape at points along a continuum of pattern shapes. This continuum is produced by the action of a group of transformations smoothly parameterized by a single variable, $s$. The procedure is to measure the visual discriminability of patterns which have been perturbed by fixed increments, $\pm \Delta s$, about $s$, as $s$ varies over its range. If there are effectively not more than two competing internal representations at each value of $s$, discrimination performance is predicted as follows. Where s is such that the assignment probabilities are maximum, discrimination performance should be minimum, and where $s$ is such that the assignment probabilities are changing most rapidly, discrimination performance should be maximum.

The experiments reported here were designed to provide some test of this approach. The plausible hypothesis was made that the variously deformed three-dot subpattern stimuli have internal representations which specify the collinearity or noncollinearity of the dots. The discriminability of pattern displays containing combinations of perturbed and unperturbed subpatterns was measured, and the form of the pattern discrimination characteristics, namely, a minimum about the in-line condition of the dots and a maximum each side, was shown to be in agreement with that predicted by the scheme.

The motivation for the use here of discrete internal representations derives from the assumption that internal representations consist of combinations of pattern attributes drawn from some fixed and finite set. The possiblity that some of the attributes have continuous values, for example, length or orientation for a line, was not considered. An alternative approach to the present experimental findings, which generalizes the notion of continuous-valued pattern attributes, supposes that patterns give rise not to discrete representations, but to unstructured fuzzy analogue representations, which vary smoothly and continuously with deformation of the pattern. [The role of internally effected transformations in an analogue system has been discussed by Foster (1978), Foster \& Mason (1979), and Shepard (in press).] The visual discriminability of two patterns within such a scheme is determined by the extent to which their fuzzy internal representations differ. This difference is then specified by some appropriate distance function. It seems unlikely that any single monotonic increasing function of the deformation of the threedot subpattern could produce the observed sharp rise and fall in the discrimination characteristics, ${ }^{6}$ but a combination of two monotonic functions of pattern deformation could suffice. Andrews et al. (1973), Ludvigh (1953) and Westheimer and McKee (1977) have shown, for various stimulus forms, that foveal spatial sensitivity (inverse threshold) for changes in pattern arrangement may show an inverted U-shaped dependence on some pattern parameters. Two suitable cues for the three-dot subpatterns would be the change in distance between the two outer dots and the mean value of their separation (compare Westheimer and McKee's Experiment 5). To test the significance of this interpretation for the present data, a control experiment was performed in which the deformation angle was fixed (at its mean magnitude of $15 \mathrm{deg}$ ) and the size of the subpattern was systemmatically varied. The subpattern was perturbed by increasing or decreasing its size about reference values in such a way that the linear displacement of the two outer dots equaled the maximum value used 
in Experiment 1. Apart from the change in parameterization, this experiment was the same as Experiment 1. The prediction of the two-cue interpretation is that discrimination performance should show an invertedU-shaped dependence on the size parameter. In fact, discrimination performance was found not to differ significantly from chance levels $(p>.1)$ for subpattern sizes encompassing those of Experiment 1. It is evidently the change in shape of the subpatterns produced by the angular perturbation, not the change in some linear dimension, which provides the key discriminatory factor.

In the analysis of the results of Experiments 1 and 2, the main concern was with the form of the discrimination characteristics. If the proposed interpretation is correct, the values of the tranformation parameter at which maximum and minimum performance occur can yield more specific information about the internal representations of the dot patterns. Thus, it appears that the internal representation specifying the noncollinearity of the dots has maximum probability of assignment when the angular deformation of the subpattern approaches $30 \mathrm{deg}$, and between 10 and $20 \mathrm{deg}$ the assignment probabilities of this and the competing representation specifying dot collinearity are changing most rapidly. These critical values of the deformation angle are, however, special to the size and density of the displays used here. For other arrangements of the subpatterns, different discrimination characteristics may be expected, and for sufficiently large deformation angles, additional internal representations should occur.

\section{REFERENCE NOTE}

1. Rumelhart, D. E. A multicomponent theory of confusion among briefly exposed alphabetic characters (Technical Report 22). San Diego: University of California, Center for Human Processing, 1971.

\section{REFERENCES}

Anrews, D. P., Butcher, A. K., \& Buckley, B. R. Acuities for spatial arrangement in line figures: Human and ideal observers compared. Vision Research, 1973, 13, 599-620.

Barlow, H. B., Narasimhan, R., \& Rosenfeld, A. Visual pattern analysis in machines and animals. Science, 1972, 177, $567-575$.

BECK, J., \& Schwartz, T. Vernier acuity with dot test objects. Vision Research, 1979, 19, 313-319.

Blesser, B., Shillman, R., Cox, C., Kuklinski, T., Ventura, J., \& EDEN, M. Character recognition based on phenomenological attributes. Visible Language, 1973, 7, 209-223.

Bouma, H., \& Andriessen, J. J. Perceived orientation of isolated line segments. Vision Research, 1968, 8, 493-507.

Broadbent, D. E. Decision and stress, London: Academic Press, 1971.

CAELLI, T. M., \& UMANSKy, J. Interpolation in the visual system. Vision Research, 1976, 16, 1055-1060.

Cox, D. R. The analysis of binary data. London: Methuen, 1970.

Foster, D. H. An approach to the analysis of the underlying structure of visual space using a generalized notion of visual pattern recognition. Biological Cybernetics, 1975, 17, 77-79.
Foster, D. H. Visual pattern recognition by assignment of invariant features and feature relations. Optica Acta, 1977, 24, 147-157.

Foster, D. H. Visual apparent motion and the calculus of variations. In E. L. J. Leeuwenberg \& H. F. J. M. Buffart (Eds.), Formal theories of visual perception. Chichester: Wiley, 1978.

Foster, D. H., \& Mason, R. J. Transformation and relationalstructure schemes for visual recognition: Two models tested experimentally with rotated random-dot patterns. Biological Cybernetics, 1979, 32, 85-93.

GiBSON, E. J. Principles of perceptual learning and development. New York: Appleton-Century-Crofts, 1969.

Liberman, A. M., Harris, K. S., Hoffman, H. S., \& Griffith, B. C. The discrimination of speech sounds within and across phoneme boundaries. Journal of Experimental Psychology, 1957, 54, 358-368.

Liberman, A. M., Harris, K. S., Kinney, J. A., \& Lane, H. The discrimination of relative onset-time of the components of certain speech and nonspeech patterns. Journal of Experimental Psychology, 1961, 61, 379-388.

Lunvigh, E. Direction sense of the eye. American Journal of Ophthalmology, 1953, 36, 139-143.

MARr, D. Early processing of visual information. Philosophical Transactions of the Royal Society, 1976, B275, 483-519.

Massaro, D. W. Auditory information processing. In W. K. Estes (Ed.), Handbook of learning and cognitive processes, $I V$. Hillsdale, N.J: Erlbaum, 1976.

Neisser, U. Cognitive psychology. New York: Appleton-CenturyCrofts, 1967.

Palmer, S. E. Structural aspects of visual similarity. Memory \& Cognition, 1978, 6, 91-97.

Pisoni, D. B., \& Lazarus, J. H. Categorical and noncategorical modes of speech perception along the voicing continuum. Journal of the Acoustical Society of America, 1974, 55, 328-333.

Prinzmetal, W., \& Banks, W. P. Good continuation affects visual detection. Perception \& Psychophysics, 1977, 21, 389-395.

PRYTULak, L. S. Good continuation revisited. Journal of Experimental Psychology, 1974, 102, 773-777.

REED, S. K. Psychological processes in pattern recognition. New York: Academic Press, 1973.

REED, S. K. Structural descriptions and the limitations of visual images. Memory \& Cognition, 1974, 2, 329-336.

ShePard, R. N. Psychophysical complementarity. In M. Kubovy \& J. R. Pomerantz (Eds.), Perceptual organization. Hillsdale, N.J.: Erlbaum, in press.

Sperling, G. A model for visual memory tasks. Human Factors, $1963,5,19-31$.

Sutherland, N. S. Outlines of a theory of visual pattern recognition in animals and man. Proceedings of the Royal Society, 1968, B171, 297-317.

Suthe RLAND, N. S. Object recognition. In E. C. Carterette \& M. P. Friedman (Eds.), Handbook of perception III. New York: Academic Press, 1973.

Turvey, M. T. Visual processing and short-term memory. In W. K. Estes (Eds.), Handbook of learning and cognitive processes V. Hillsdale, N.J: Erlbaum, 1978.

UtTal, W. R. The effect of deviations from linearity on the detection of dotted line patterns. Vision Research, 1973, 13, 2155-2163.

WERTHEIMER, M. Untersuchungen zur Lehre von der Gestalt, II. Psychologische Forschung, 1923, 4, 301-350.

Westheimer, G., \& McKeE, S. P. Spatial configurations for visual hyperacuity. Vision Research, 1977, 17, 941-947.

\section{NOTES}

1. The lower unperturbed subpatterns in Figures $3 a$ and $3 b$ were included in the display to raise the discrimination threshold above that for conventional vernier acuity. 
2. The dots, which were white, appeared bright on a uniform 9-deg square white background field, luminance $100 \mathrm{~cd} \mathrm{~m}^{-2}$. The subject set the luminous intensity of the dots (typically $50 \mu \mathrm{cd} \mathrm{sec)}$ so that when the dots were flashed for the duration used in the experiment they were 10 times above increment threshold.

3. The individual dots comprising each subpattern were clearly resolvable at the spatial separations used in the experiments. By means of a $2 \mathrm{AFC}$ discrimination task, dot separation was shown to be at least twice the minimum resolvable. The fidelity of the display was such that deviations of the dots from their correct locations produced less than $5 \%$ error in the increment $\Delta \mathrm{s}$.

4. Only one of the two sets of data for subject F.M.F. were required for the analysis, and the set corresponding to the larger magnitude of $\Delta \mathrm{s}$ and the higher discrimination performance was omitted.

5. The significant result for an asymmetry in discrimination performance about $s=0 \mathrm{deg}$ implies, within the present scheme, a corresponding (small) asymmetry in the rates of falloff in representation assignment probability with pattern deformation.

6. A plausible distance function is the linear separation of the two outer dots of the subpattern. The changes in this distance with changes $\Delta s$ in angle $s$ do not, however, decrease with $s$ when $s$ is large. It might be hypothesized that the change from increasing to decreasing performance with $s$ is a result of a switch from the use of one distance function to another. A combination of functions may obviously be chosen to fit the data, but this interpretation merely shifts the origin of the proposed discreteness property from the internal representations to the operations by which they are compared. See comments by Foster and Mason (1979).

(Received for publication May 8, 1979; revision accepted September 27, 1979.)

\section{APPENDIX 1}

Let $A$ be a pattern and $\psi_{s}, a \leqslant s \leqslant b, a<b$, be a local 1-parameter group of differentiable transformations. The action of $\psi_{\mathrm{s}}$ on $\mathrm{A}$ is denoted by $\psi_{\mathrm{s}}(\mathrm{A})$. The group property means that $\psi_{s+t}(\mathrm{~A})=\psi_{\mathrm{s}}\left(\psi_{\mathrm{t}}(\mathrm{A})\right)$ for all $\mathrm{s}, \mathrm{t}, \mathrm{s}+\mathrm{t}$ in the interval $[a, b]$. [More precise definitions of patterns and transformation groups may be found in Foster (1975). The points $a, b$, etc., are unrelated to those in Figure 1.]

Suppose that $\mathbf{A}$ and $\psi_{s}, \mathrm{a} \leqslant \mathrm{s} \leqslant \mathrm{b}$, are such that there is a fixed and finite family of distinct internal representations, $r_{i}, 1 \leqslant i \leqslant n(n \geqslant 2)$, which may be assigned, one at a time, to the transformed patterns, $\psi_{s}(A)$. For each $s$ in $[a, b]$, let $p_{i}\left(\psi_{s}(A)\right)=p_{i}(s)$ be the probability of internal representation $r_{i}$ being assigned to $\psi_{s}(A), 1 \leqslant i \leqslant n$. Note that $\sum_{i=1}^{n} p_{i}(s)=1$, for all $s$ in $[a, b]$. Suppose that the functions $\mathrm{p}_{\mathrm{i}}, 1 \leqslant \mathrm{i} \leqslant \mathrm{n}$, are differentiable and satisfy the conditions set out below. The first condition requires that the transformations are such that as the pattern is transformed away from the single region on which some internal representation has maximum probability of assignment there are no irregularities in the smooth falloff in this representation's probability of assignment. Maxima of different internal representation probabilities should also not overlap. The second condition requires that for adjacent representations $r_{i-1}, r_{i}, r_{i+1}$ the assignment probability of $r_{i}$ is not significant in either magnitude or relative rate of change when the pattern is transformed to or beyond the point at which $r_{i-1}$ and $r_{i+1}$ have maximum assignment probabilities. For each transformed pattern $\psi_{\mathrm{s}}(\mathrm{A})$, there are, thus, effectively at most two internal representations in competition with each other.

(1) For each function $p_{i}, 1 \leqslant i \leqslant n$, there is a single interval $\left[c_{i}, d_{i}\right], a \leqslant c_{i} \leqslant d_{i} \leqslant b$, on which $p_{i}$ achieves its maximum value, and such that on $\left[a, c_{j}\right] p_{i}$ is monotonic increasing and on $\left[\mathrm{d}_{\mathrm{i}}, \mathrm{b}\right]$ it is monotonic decreasing, with at most a single point of inflexion in each case. For all $i, 1 \leqslant i<n$, let $\mathrm{d}_{\mathrm{i}}<\mathrm{c}_{\mathrm{i}+1}$.

(2) There is a fixed quantity $\varepsilon, 0<\varepsilon \ll 1$, such that for each $p_{i}, 1 \leqslant i \leqslant n$,

$$
0 \leqslant p_{i}(s),\left|p_{i}^{\prime}(s)\right| \cdot k^{-1} \leqslant \varepsilon
$$

for $a \leqslant s \leqslant d_{i-1}, 1<i \leqslant n$, and $c_{i+1} \leqslant s \leqslant b, 1 \leqslant i<n$, where

$$
k=\min _{1 \leqslant j \leqslant n} \max _{a \leqslant s \leqslant b}\left|p_{j}^{\prime}(s)\right|
$$

and $\mathrm{p}_{i}(\mathrm{~s})$ is the derivative of $\mathrm{p}_{\mathrm{i}}$ at $\mathrm{s}$.

Suppose $s$ is restricted to the fixed interval $d_{i-1}<s \leqslant d_{i}$, for some $\mathrm{i}, 1<\mathrm{i} \leqslant \mathrm{n}$. Let the transformed pattern $\psi_{\mathrm{s}}(\mathrm{A})$ be given additional fixed incremental transformations $\psi_{-\Delta s}$ and $\psi_{\Delta s}, \Delta s>0$. By the group property of $\psi_{s}, \psi_{-\Delta s}\left(\psi_{s}(\mathrm{~A})\right)$ $=\psi_{\mathrm{s}}-\Delta \mathrm{s}(\mathrm{A})$ and $\psi_{\Delta \mathrm{s}}\left(\psi_{\mathrm{s}}(\mathrm{A})\right)=\psi_{\mathrm{s}}+\Delta \mathrm{s}(\mathrm{A})$ (providing $\mathrm{s}-\Delta \mathrm{s}$ and $s+\Delta s$ are in $[a, b])$. The visual discriminability of the two patterns, $\psi_{\mathrm{s}}-\Delta \mathrm{s}(\mathrm{A})$ and $\psi_{\mathrm{S}+\Delta \mathrm{s}}(\mathrm{A})$, is assumed to be determined by a monotonic increasing function of the differences between the assignment probabilities of the internal representations produced by the two patterns. The "distance" $e(s)$ between $\left(p_{1}(s-\Delta s), p_{2}(s-\Delta s), \ldots, p_{n}(s-\Delta s)\right)$ and $\left(\mathrm{p}_{1}(\mathrm{~s}+\Delta \mathrm{s}), \mathrm{p}_{2}(\mathrm{~s}+\Delta \mathrm{s}), \ldots, \mathrm{p}_{\mathrm{n}}(\mathrm{s}+\Delta \mathrm{s})\right)$ is quantified with the $\ell_{\mathrm{p}}^{\mathrm{n}}$ norm:

$$
\left\|\left(x_{1}, x_{2}, \ldots, x_{n}\right)\right\|=\left(\sum_{j=1}^{n}\left|x_{j}\right|^{q}\right)^{1 / q},
$$

where $q$ is fixed and $1 \leqslant q<\infty$. When $q=2$, this is the Euclidean metric, and when $q=1$, the city-block metric. Thus,

$$
\begin{aligned}
e(s)= & \|\left(p_{1}(s+\Delta s)-p_{1}(s-\Delta s), p_{2}(s+\Delta s)-p_{2}(s-\Delta s)\right. \\
& \left.\ldots, p_{n}(s+\Delta s)-p_{n}(s-\Delta s)\right) \|
\end{aligned}
$$

Making use of Taylor's formula and ignoring terms of the order $\varepsilon$ and $(\Delta s)^{3}$ and higher, one obtains the result that, for sufficiently small values of $\Delta s$,

$$
e(s)=2^{(1+q) / q} \cdot \Delta s \cdot\left|p_{i}^{\prime}(s)\right| ;
$$

the function e has a maximum when $\left|p_{i}^{\prime}\right|$ has a maximum, and a minimum when $p_{i}$ has a maximum.

Note that the qualitative characteristics of $e$ (positions of extrema and inflexion points) do not depend on the choice of $\ell_{p}^{n}$ metric. Because of the approximations involved in deducing the above expression, the minimum value of e when computed accurately will in general be nonzero. Note also that the requirement that $\Delta s$ be "sufficiently small" is quantifiable only when the $p_{i}$ are specified more precisely. When $\Delta s$ is not sufficiently small, the minimum value of $e$ need no longer occur where $p_{i}$ has its maximum. One way to verify that the magnitude of $\Delta$ s chosen experimentally is not 
too large is to show that reducing $\Delta$ s does not affect the form of the discrimination characteristics (compare results of main and auxiliary parts of Experiment 2).

\section{APPENDIX 2}

The following statistical technique, based on the use of the logistic transform $\log [p /(1-p)]$ of the probability $p$, is taken from Cox (1970).

Let there be $k$ sets of observations. In each set $j$, let the probability of success be $\pi_{j}$, the number of trials be $n_{j}$, and the number of successes be $t_{j}$; let $\lambda_{j}$ be the logistic transform of $\pi_{j}$. Suppose we wish to test the null hypothesis that

$$
\Sigma_{j} c_{j} \lambda_{j}=0,
$$

where the $c_{j}$ are constants. Let

$$
u_{j}=\log \left[\left(t_{j}+1 / 2\right) /\left(n_{j}-t_{j}+1 / 2\right)\right],
$$

and

$$
v_{j}=\frac{\left(n_{j}+1\right)\left(n_{j}+2\right)}{n_{j}\left(t_{j}+1\right)\left(n_{j}-t_{j}+1\right)} .
$$

Then, if the null hypothesis is true, the contrast

$$
\frac{\Sigma_{j} c_{j} u_{j}}{\sqrt{\Sigma_{j} c_{j}^{c} v_{j}}}
$$

is distributed approximately as the standard normal variable $z$. It follows that the sum of squares of $m$ such contrasts is distributed approximately as chi squared with $\mathrm{m}$ degrees of freedom, providing the contrasts are independent. 